\title{
Mode-field matching design, 3D fabrication and characterization of down-tapers on single-mode optical fiber tips for coupling to photonic integrated circuits
}

Vanmol, Koen, Saurav, Kumar, Panapakkam, Vivek, Van Thourhout, Dries, Thienpont, Hugo, et al.

Koen Vanmol, Kumar Saurav, Vivek Panapakkam, Dries Van Thourhout, Hugo Thienpont, Nathalie Vermeulen, Jan Watté, Jürgen Van Erps, "Mode-field matching design, 3D fabrication and characterization of down-tapers on single-mode optical fiber tips for coupling to photonic integrated circuits," Proc. SPIE 11349, 3D Printed Optics and Additive Photonic Manufacturing II, 1134907 (30 March 2020); doi: 10.1117/12.2555770 


\title{
Mode-field matching design, 3D fabrication and characterization of down-tapers on single-mode optical fiber tips for coupling to photonic integrated circuits.
}

\author{
Koen Vanmol*a, Kumar Saurav ${ }^{\mathrm{b}}$, Vivek Panapakkam ${ }^{\mathrm{b}}$, Dries Van Thourhout ${ }^{\mathrm{c}}$, \\ Hugo Thienpont ${ }^{\mathrm{a}}$, Nathalie Vermeulen ${ }^{\mathrm{a}}$, Jan Watté ${ }^{\mathrm{b}}$, Jürgen Van Erps ${ }^{\mathrm{a}}$ \\ ${ }^{a}$ Brussels Photonics (B-PHOT), Dept. of Applied Physics and Photonics, Vrije Universiteit Brussel \\ and Flanders Make, Pleinlaan 2, 1050 Brussels, Belgium; ${ }^{b}$ CommScope, Diestsesteenweg 692, 3010 \\ Leuven, Belgium; 'Dept. of Information Technology (INTEC), Ghent University-IMEC, \\ Technologiepark-Zwijnaarde 126, 9000 Gent, Belgium
}

\begin{abstract}
Photonic Integrated Circuits have made it possible to decrease the footprint of traditionally bulky optical systems and they create opportunities for various new and fascinating applications. One of the limiting factors for the widespread adaption of PICs is their connection to the outside world. As the mode field diameter of optical modes in waveguides tends to be an order of magnitude smaller than in their fiber counterparts, creating an efficient, robust and alignmenttolerant fiber-to-chip interface remains a challenge.

In this work, we investigate the optimization of the fiber-side of the optical interface, whereas the chip itself remains untouched and makes use of spot-size convertors. Optical fiber tips can be functionalized using two-photon polymerization-based 3D nanoprinting technology, which offers full 3D design freedom and sub-micrometer resolution. We present a down-taper design strategy to match the mode-field diameter of single-mode optical fibers to the modefield diameter of waveguides with spot-size converters on PICs.

The 3D printed down-tapers are characterized towards their geometry and mode shape, and we experimentally demonstrate their use for coupling towards a Silicon-On-Insulator chip with spot-size convertors. Furthermore, the performance of these down-tapered fibers is compared to conventional lensed fibers in terms of optical coupling efficiency.
\end{abstract}

Keywords: 3D direct laser writing, fiber-to-chip coupling, fiber taper, mode-matching, optical interconnects, photonic integrated circuits, two-photon polymerization

\section{INTRODUCTION}

The integration of many different optical components and functionalities into a compact device or chip, the so-called Photonic Integrated Circuit (PIC), has been actively investigated for already many years. Up to now, the silicon materialplatform is the predominantly used platform with applications ranging from telecommunications and interconnects to onchip sensors ${ }^{1,2}$. The main reason for the widespread use of the silicon-platform is the fact that it can make use of highyield optimized processing techniques compatible with the complementary metal-oxide-semiconductor (CMOS) manufacturing industry. As such, high-volume fabrication of small-footprint chips with silicon-on-insulator (SOI) waveguides is readily available.

Whereas the many developments in chip-level components bring various fascinating opportunities and applications, one of the limiting factors in the widespread adoption of PICs is their packaging, and in particular their connection to the outside world. As can be seen from Figure 1, SOI waveguides tend to be an order of magnitude smaller than the core of a standard single-mode fiber (SMF), and so is the case for the optical modes. This large difference in physical dimensions severely reduces the coupling efficiency between both, where less than $1 \%$ of the light would be coupled, leading to a loss of more than $20 \mathrm{~dB}$ per facet. Because of this, creating a highly efficient, robust and alignment-tolerant fiber-to-chip interface remains a challenge.

3D Printed Optics and Additive Photonic Manufacturing II, edited by Alois M. Herkommer, Georg von Freymann, Manuel Flury, Proc. of SPIE Vol. 11349, 1134907 - @ 2020 SPIE $\cdot$ CCC code: $0277-786$ X/20/\$21 · doi: $10.1117 / 12.2555770$ 

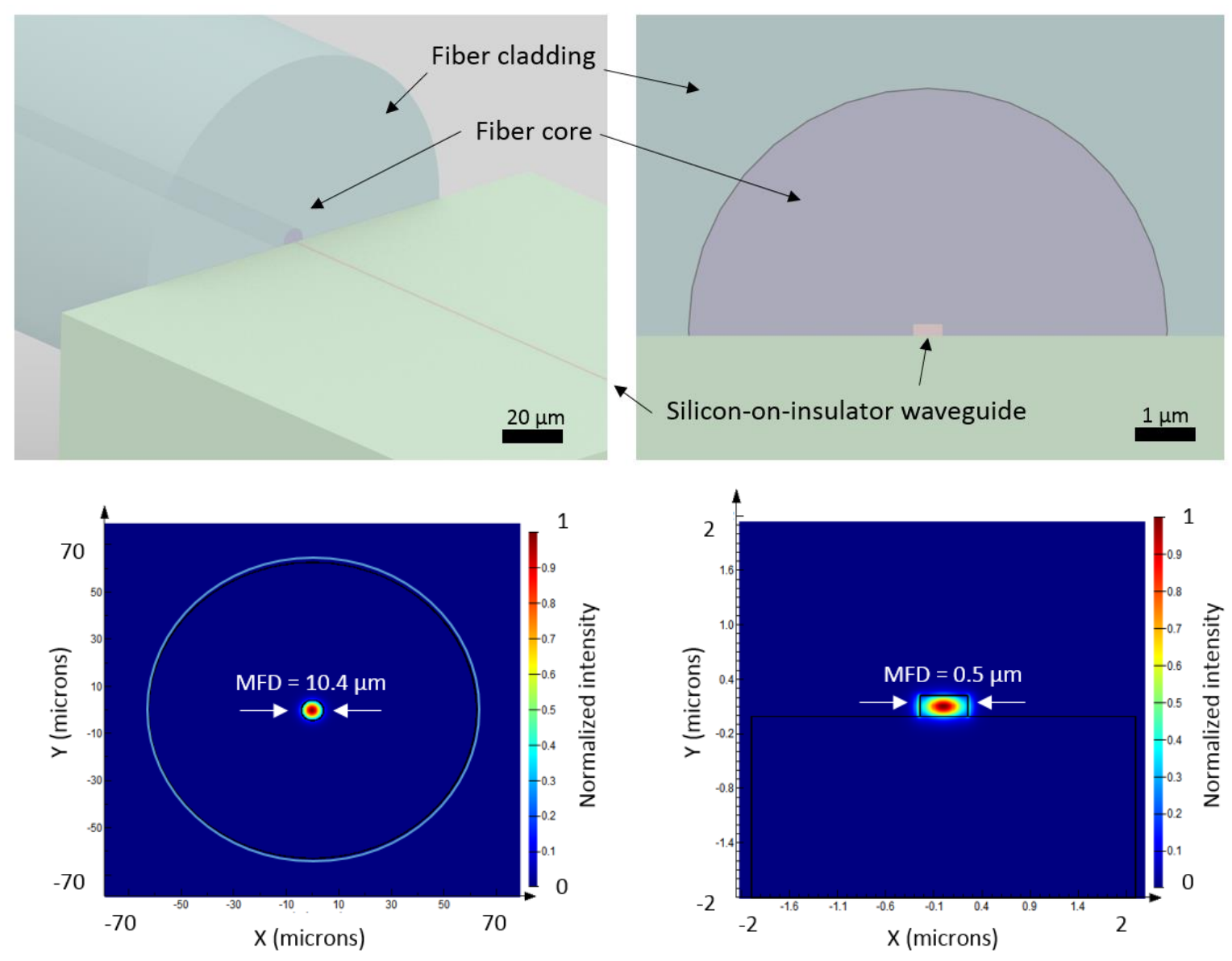

Figure 1. Top: difference in physical dimensions between a single-mode optical fiber and a silicon-on-insulator waveguide. Bottom: Simulations showing the difference in size between the propagating modal fields in a single-mode optical fiber and a silicon-on-insulator waveguide. The un-matched mode-field diameters (MFD) would lead to large coupling losses.

In order to overcome these direct coupling losses, two main coupling strategies are actively investigated in research, being grating couplers and edge couplers. These coupling schemes are schematically presented in Figure 2 . Vertical coupling through the use of on-chip diffractive grating structures (Figure 2(a)) offers the possibility of multipoint wafer characterization and shows relatively relaxed fiber positioning tolerances. A disadvantage of these diffractive structures is that they are wavelength and polarization dependent, making them unsuitable for certain applications.

A second type of coupling scheme is the in-plane or edge coupler. This approach generally offers the best optical performance in terms of coupling efficiency, spectral transmission bandwidth and polarization independency ${ }^{3,4}$, and is therefore of high interest. The edge coupling technique often relies on an on-chip coupling structure (usually some kind of taper) that transforms and matches the modal field of the waveguide to that of the SMF (which is often a specialty fiber). The most frequently used types of specialty fibers for fiber-to-chip coupling are lensed fibers ${ }^{5}$ (Figure 2(b)) that produce a focal spot around 2-3 $\mu \mathrm{m}$ in diameter. This free-space approach of coupling will always be affected by Fresnel reflections at the optical interfaces and is limited in its design freedom on the shape and size of the focused spot, making it hard to exactly match the chip's modal fields. 
(a)

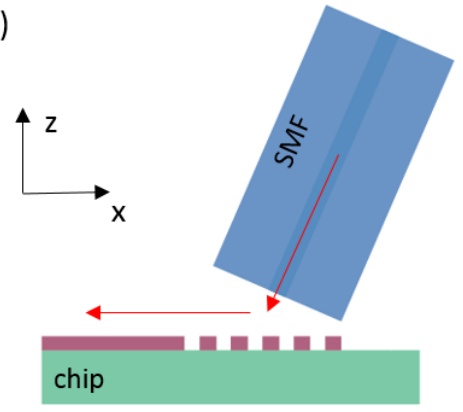

(b)

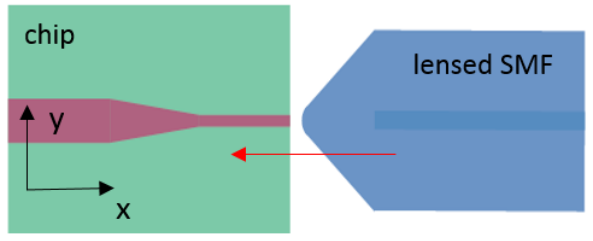

(c)

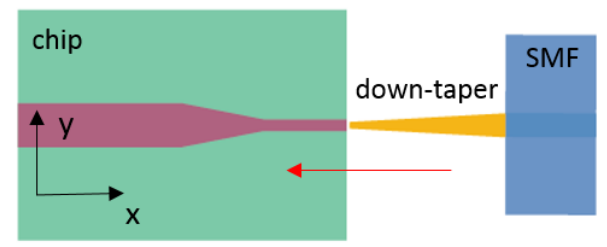

Figure 2. Coupling schemes between a single-mode optical fiber and a silicon-on-insulator chip. (a) Grating coupling scheme, (b) edge coupling scheme with a lensed optical fiber, and (d) proposed edge coupling scheme with a 2PP printed down-taper on the facet of an SMF. The red arrows indicate the direction of the in-coupled light. Dimensions are not to scale.

As many different types of on-chip coupling schemes are still being actively explored ${ }^{6}$, our design strategy is to optimize the fiber's side of the fiber-to-chip connection and tailor the design of the fiber taper to the on-chip waveguide schemes being employed by the PIC foundry.

We propose to functionalize SMF end-facets with down-taper structures by means of two-photon polymerization (2PP)based direct laser writing (Figure 2(c)). In this technology, femtosecond laser pulses focused through a high-numerical aperture microscope objective initiate the two-photon absorption process to polymerize a photosensitive material in a localized focal spot. By scanning the laser beam in a layer-by-layer fashion, we are therefore able to fabricate 3D structures with full design freedom and submicrometer resolution ${ }^{7}$.

In recent years, 3D nanoprinting technology, based on two-photon polymerization, is driving advancements in photonic miniaturization and integration. Research in this topic has shown that optical fibers can be functionalized for many applications, where the 2PP additive manufacturing technique has already been used in the development of a microstructured antireflective coating ${ }^{8}$, phase masks ${ }^{9}$, lenses ${ }^{10,11}$ and mode-field expansion up-taper structures ${ }^{12}$ on optical fiber tips. In the latter, we demonstrated the fabrication method for use in alignment tolerant physical contact expanded beam fiber-to-fiber connections.

Recently, next to the 2PP printed free-space fiber-to-chip configurations targeted by Dietrich et al ${ }^{13}$, photonic wire bonds developed at the Karlsruhe Institute of Technology (KIT) have shown a lot of opportunities with the demonstration of hybrid photonic integration using 2PP-printed permanent interconnections. As such, permanent single-core and multicore optical fiber connections towards Si-chips ${ }^{14}$ and connections between InP-based lasers and Si-chips ${ }^{15}$ have been established. Such permanent connections have very high potential in reducing the cost of active alignment strategies, but still have several challenges to overcome. Highly accurate (submicrometer) positioning is needed as tight alignment tolerances are observed for SOI chips. Optimal coupling in a passive way is therefore very hard to obtain.

In this paper, we investigate the design and $2 \mathrm{PP}$ fabrication of down-taper structures on single-mode optical fibers that could overcome the above-mentioned challenges. Our target is to establish physical contact connections (to reduce reflections) without the need for physical changes to the chips, as they are usually still rather costly and available in only a small amount. We optimize the taper geometry for adiabatic transmission by simulating the light propagation with mode solving software. Afterwards, the fabricated tapers on fiber tips are characterized experimentally in terms of coupling loss. 


\section{DOWN-TAPER DESIGN}

In our design, a single-mode fiber is tapered down to a smaller cross section. This is done by 3D printing of a downtapered core structure on the cleaved facet of the fiber. In this configuration no cladding material is present and therefore, even though the core diameter is decreased, the structure becomes highly multi-mode. As the different modes can exchange energy between each other, it is possible that part of this energy gets radiated out of the waveguide, giving rise to radiation loss. However, mode coupling to higher order modes can be avoided by careful design of the taper structure. To ensure minimal loss, it is therefore desired to have a maximum transmission of the fundamental mode. In general, the more gradual the change in cross section of the taper is, the better the transmission of this fundamental mode becomes ${ }^{16}$. In this paper we will therefore make use of a gradually changing (adiabatic) linear taper structure.

We used the Lumerical Mode Solutions software ${ }^{17}$ to model the light propagation through a tapered fiber. This eigenmode solver calculates several sets of supported mode profiles at different cross-sectional locations of our taper geometry using a finite mesh. Mode coupling coefficients are then calculated between these different sets and a full scattering matrix is obtained that gives the transmission and reflection coefficients for all supported modes inside the device. Afterwards, little additional computation is needed to sweep the taper length and find its optimal value to achieve the highest adiabatic transmission.

We chose to make use of a standard G. $652^{18} \mathrm{SMF}$ and the commercially available and optimized IP-DIP photoresist formulation from Nanoscribe $\mathrm{GmbH}^{19}$. The Mode Solutions software makes it possible to find the optimal taper input diameter for maximal mode overlap with the SMF and the mode-matched taper output diameter for optimal coupling towards the SOI waveguide, which makes use of an on-chip spot-size convertor with a mode-field diameter of about 3 to $4 \mu \mathrm{m}$. Sweeping the taper length allows us to analyze the transmission of the fundamental mode, as shown in Figure 3. This way, we found that a taper length of about $243 \mu \mathrm{m}$ would be adiabatic enough to ensure that the output modal field closely matches the waveguide mode.
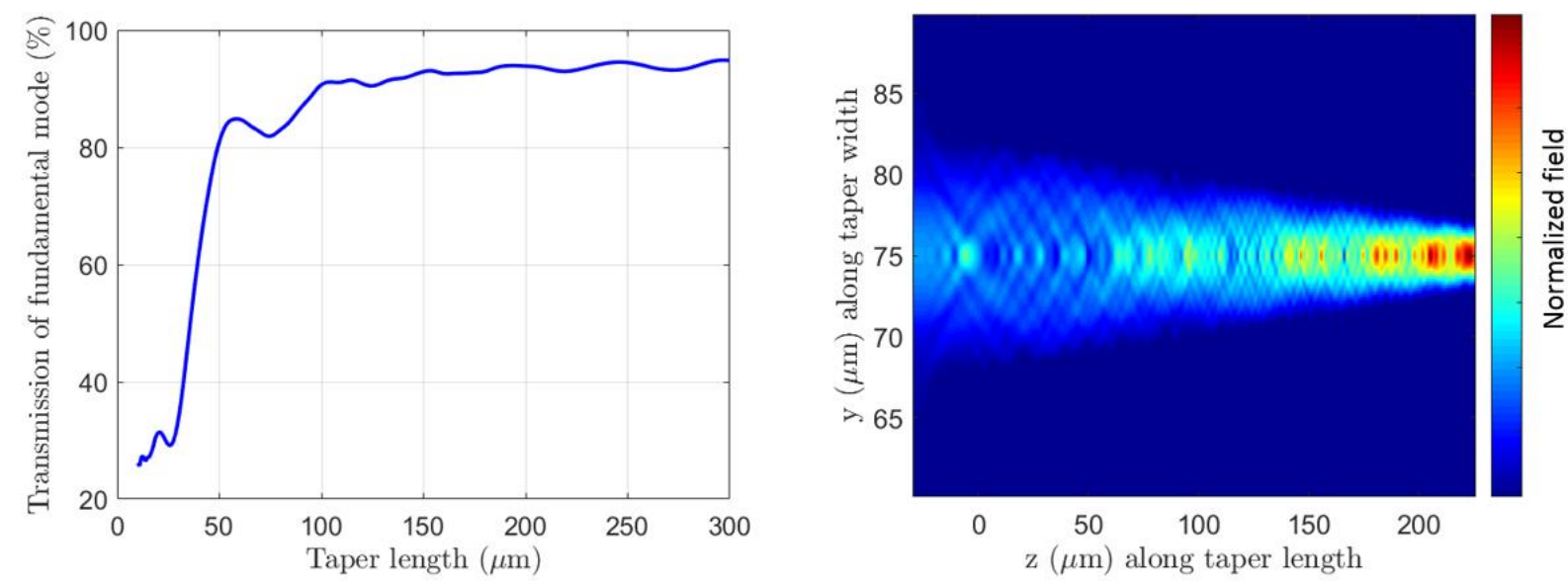

Figure 3. Simulation results for the down-tapered optical fiber. Left: Transmission of the fundamental mode as a function of the taper length. Right: Field propagation along the down-taper. Simulated at $1550 \mathrm{~nm}$. 


\section{3-DIMENSIONAL DIRECT LASER FABRICATION}

From the simulations, an optimal geometry was obtained for our linear down-taper. For the printing job we used the commercial Photonic Professional GT+ system from Nanoscribe GmbH together with IP-DIP photoresist material ${ }^{19}$. By making use of an external red light source connected to the fiber, we are able to image the fiber's core before printing. In the manufacturing procedure, the built-in CCD camera makes it then possible to focus on the fiber facet and align to the illuminated core. The computer-aided design and manufacturing software from Nanoscribe (Describe and Nanowrite) is then used to transfer the designed component into the photoresist droplet by direct laser writing.

Regarding the fabrication of a polymer taper structure with high aspect ratio, i.e. a long length compared to its bottom diameter, good adhesion to the fused silica $\left(\mathrm{SiO}_{2}\right)$ end facet of the cleaved fiber is crucial. That is why we are adding a thin base plate layer underneath the taper structure, to increase the effective contact area between the polymer taper and glass fiber.

After fabrication, the printed structure is developed using propylene glycol methyl ether acetate developer (PGMEA) and we obtain a free-standing air-clad taper core. A scanning electron microscope image of the final result is shown in Figure 4. The physical dimensions of the taper-structure were verified to be within a $1 \mu \mathrm{m}$ tolerance of the designed structure using a Bruker Contour GT-I white-light interferometry system and a Werth VideoCheck UA400 coordinate measurement machine.

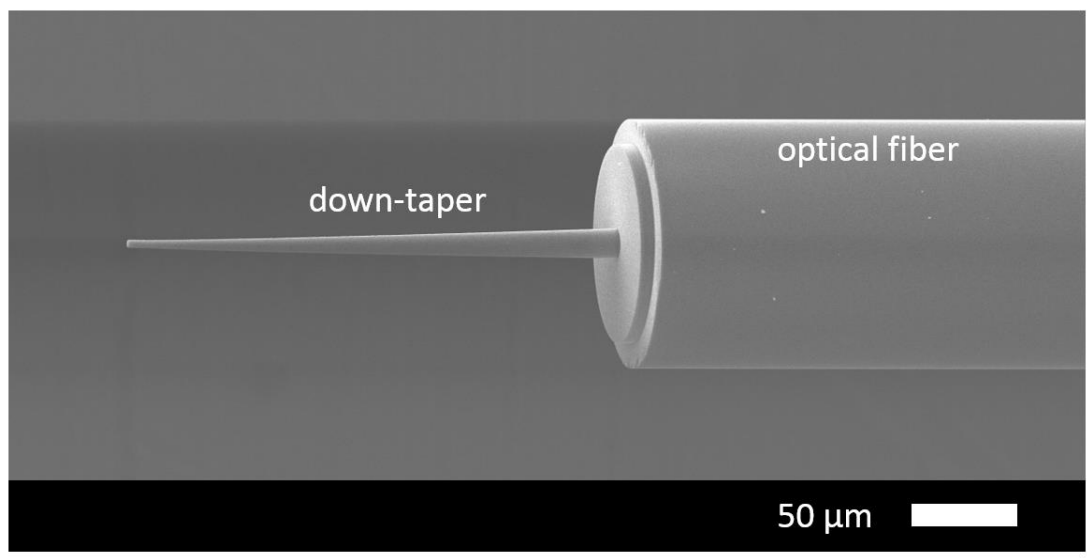

Figure 4. Scanning electron microscope image of a 3D printed down-taper structure on top of a cleaved optical fiber tip.

\section{OPTICAL CHARACTERIZATION}

Two of these fabricated down-tapered fibers were used in coupling experiments with the SOI chip. The mode-field diameter of the down-tapers, measured with an infrared-sensitive microscope to be around $3.8 \mu \mathrm{m}$, matches very well with the modal fields of the on-chip spot-size converters. In addition, Fresnel reflections are limited by making sure physical contact is achieved. Because of the optimal design, a very good coupling efficiency of $1.34 \mathrm{~dB}$ per facet was experimentally obtained. To compare, we used commercially available lensed fibers, which have an MFD of about 2.8 $\mu \mathrm{m}$, and measured the coupling loss in the same way. For these lensed fibers a coupling loss of $2.31 \mathrm{~dB}$ per facet is measured. As such, it is clear that an optimization towards modal overlap of the fiber and waveguide, together with the physical contact approach can drastically improve the coupling efficiency.

All measurements are done without the help of index matching gel, as this could be seen as a cladding material for the taper, altering its transmission characteristics. In a later stage, the taper design will be adapted to include an index matching gel or liquid around the tapered core. This way, the influence of Fresnel reflections can be further reduced. 


\section{CONCLUSION}

We have demonstrated the design and 2PP fabrication of 3D nanoprinted down-taper structures on top of cleaved optical fiber tips to match the mode profile of the fiber to that of the SOI chip. Mode solver simulations make it possible to maximize the modal overlap with the chip and reduce higher-order mode coupling in the taper, which makes this design strategy very flexible as it can be adapted for different types of fibers, photoresist materials and desired mode-field diameters. Coupling measurements show that good overlap is achieved between the fiber and waveguide modes, where the coupling loss per facet was measured to be only $1.34 \mathrm{~dB}$. The coupling efficiency with the $2 \mathrm{PP}$ down-tapers was compared with commercially available lensed fibers, for which a coupling loss of $2.31 \mathrm{~dB}$ per facet was obtained. It is clear, that both the modal field design freedom and the physical contact approach allowed by our down-taper structures are effectively beneficial to achieve best coupling efficiencies.

\section{ACKNOWLEDGMENTS}

This work was financially supported by the Flemish Agency for Innovation and Entrepreneurship (VLAIO) under contract number IWT.150924 (FANTOAM) and in part by Interreg NWE758, BELSPO-IAP, IOF, Hercules/FWO $\mathrm{UAB} / 13 / 10$ and the Methusalem foundations, and by the OZR of the Vrije Universiteit Brussel.

\section{REFERENCES}

[1] P. Dong, Y. Chen, G. Duan, and D. Neilson, "Silicon photonic devices and integrated circuits," Nanophotonics, 2015.

[2] J. E. Bowers, T. Komljenovic, M. Davenport, J. Hulme, A. Y. Liu, C. T. Santis, A. Spott, S. Srinivasan, E. J. Stanton, and C. Zhang, "Recent advances in silicon photonic integrated circuits," in Next-Generation Optical Communication: Components, Sub-Systems, and Systems V, vol. 9774, 2016, pp. 1-18.

[3] G. Son, S. Han, J. Park, K. Kwon, and K. Yu, "High-efficiency broadband light coupling between optical fibers and photonic integrated circuits," Nanophotonics, 2018.

[4] R. Marchetti, C. Lacava, K. Gradkowski, and P. Minzioni, "Coupling strategies for silicon photonics integrated chips," Photonics Research, vol. 7, no. 2, 2019.

[5] H. M. Presby and C. A. Edwards, "Near 100\% efficient fiber microlenses," Electron. Lett., vol. 28, no. 6, 1992.

[6] X. Mu, S. Wu, L. Cheng, and H. Fu, "Edge Couplers in Silicon Photonic Integrated Circuits : A Review," Applied Sciences, 2020.

[7] M. Farsari and B. N. Chichkov, "Materials processing: Two-photon fabrication," Nature Photonics, vol. 3, no. 8, pp. 450-452, 2009.

[8] Kowalczyk, M., Haberko, J., Wasylczyk, P., "Microstructured gradient-index antireflective coating fabricated on a fiber tip with direct laser writing," Optics Express 22 (10), 12545-12550 (2014).

[9] Gissibl, T., Schmid, M., Giessen, H., "Spatial beam intensity shaping using phase masks on single-mode optical fibers fabricated by femtosecond direct laser writing," Optica 3 (4), 448-451 (2016).

[10] Gissibl, T., Thiele, S., Herkommer, A., Giessen, H., "Two-photon direct laser writing of ultracompact multilens objectives," Nature Photonics 10 (8), 554-560 (2016).

[11] Gissibl, T., Thiele, S., Herkommer, A., Giessen, H., "Sub-micrometre accurate free-form optics by threedimensional printing on single-mode fibres," Nature Communications 7.

[12] K. Vanmol, S. Tuccio, V. Panapakkam, H. Thienpont, J. Watté, and J. Van Erps, "Two-photon direct laser writing of beam expansion tapers on single-mode optical fibers," Optics and Laser Technology, vol.112, pp. 292-298, 2019.

[13] Dietrich, P. I., Blaicher, M., Reuter, I., Billah, M., Hoose, T., Hofmann, A., Caer, C., Dangel, R., Offrein, B., Troppenz, U., Moehrle, M., Freude, W. and Koos, C., "In situ 3D nanoprinting of free-form coupling elements for hybrid photonic integration," Nature Photonics 12 (4) (2018) 241-247.

[14] Lindenmann, N., Dottermusch, S., Goedecke, M. L., Hoose, T., Billah, M. R., Onanuga, T. P., Hofmann, A., Freude, W., Koos, C., "Connecting silicon photonic circuits to multicore fibers by photonic wire bonding," Journal of Lightwave Technology 33(4), 755-760 (2015). 
[15] M. Billah, M. Blaicher, T. Hoose, P. Dietrich, P. Marin-Palomo, N. Lindenmann, A. Nesic, A. Hofmann, U. Troppenz, M. Moehrle, S. Randel, W. Feude, and C. Koos, "Hybrid integration of silicon photonics circuits and InP lasers by photonic wire bonding," Optica, vol. 5, no. 7, 2018.

[16] Snyder, A., Love, J., [Optical Waveguide Theory], Springer US (1983).

[17] Lumerical <http://www.lumerical.com>

[18] International Telecommunication Union 2009 Recommendation G.652 - Characteristics of a single-mode optical fibre and cable (2009).

[19] Nanoscribe GmbH <https://www.nanoscribe.de/en/> 